\title{
Verbraucher- und Gläubigerrechte in der Corona-Krise - Ausweitung oder Einschränkung?
}

\author{
Dr. Caspar Behme"
}

\section{Inhaltsübersicht}

A. Pandemiebedingte Leistungsstörungen und gesetzliche Risikoverteilung $\quad 74$

I. Dauerhafte Unmöglichkeit der Leistungserbringung 74

1. Befreiung der Parteien von ihren wechselseitigen Leistungspflichten und Rücktrittsrecht des Gläubigers

2. Schadensersatzpflicht des Schuldners $\quad 77$

II. Verzögerung der Leistungserbringung $\quad 78$

1. Zurückbehaltungsrecht und Rücktrittsrecht des Gläubigers 78

2. Schadensersatzpflicht des Schuldners $\quad 79$

III. Vorübergehende Unmöglichkeit der Leistungserbringung 79

1. Befreiung der Parteien von ihren wechselseitigen Leistungspflichten und Rücktrittsrecht des Gläubigers $\quad 80$

2. Schadensersatzpflicht des Schuldners 81

IV. Unzumutbarkeit der Leistungserbringung 82

V. Zwischenfazit: Gesetzliche Zuweisung des Risikos pandemiebedingter
Leistungsstörungen an den Schuldner

B. Auswirkungen der gesetzlichen Risikoverteilung $\quad 85$

I. Fallbeispiel 1 - Lieferkette $\quad 85$

II. Fallbeispiel 2: Abgesagtes Konzert $\quad 87$

III. Fallbeispiel 3: Lieferung einer mangelhaften Sache $\quad 88$

C. Zwischenfazit und rechtsökonomische Bewertung 88

D. Alternativvorschlag: Zuweisung des Risikos pandemiebedingter Leistungsstörungen an den Gläubiger

I. Auswirkungen auf Fallbeispiel 1

II. Auswirkungen auf Fallbeispiel $2 \quad 92$

III. Ein letzter Blick auf Fallbeispiel 3

Unternehmen, die infolge der Corona-Pandemie in eine wirtschaftiche Schieflage geraten, sind in der Regel in ihrer Rolle als Schuldner betroffen: Weil in den vertraglichen Beziehungen mit ibren Kunden Leistungsstörungen eintreten, deren Risiko sie nach der gesetzlichen Risikoverteilung zu tragen haben, verlieren sie ihre Vergütungsansprüche oder ihr Vertragspartner

* Der Verfasser ist Habilitand an der LMU München und Vertretungsprofessor für Bürgerliches Recht an der Universität Bremen. 
kann sich vollständig vom Vertrag lösen. Der nachfolgende Beitrag erläutert einige typische Fallbeispiele und ihre Behandlung nach geltendem Recht. Im Anschluss daran wird ein alternatives Lösungsmodell vorgestellt, in dem das Risiko pandemiebedingter Leistungsstörungen dem Gläubiger zugewiesen wird. Anhand der behandelten Fallkonstellationen wird sodann aufgezeigt, dass dieses Modell regelmäßig zu sach- und interessengerechten Lösungen fübren wird.

\section{A. Pandemiebedingte Leistungsstörungen und gesetzliche Risikoverteilung}

In zahlreichen vertraglichen Beziehungen führt die Corona-Pandemie zu Situationen, die das bürgerliche Recht als „Leistungsstörungen“ bezeichnet. Hinsichtlich der infolge der Auswirkungen der Corona-Pandemie denkbaren Leistungsstörungen ist zwischen Fällen zu differenzieren, in denen die Erbringung der Leistung für den Schuldner unmöglich wird (unter 1.), und solchen, in denen sich die Erbringung der Leistung lediglich verzögert (unter 2.). Gewissermaßen zwischen diesen Szenarien liegen Fälle, in denen die Leistung dem Schuldner vorübergehend unmöglich ist (unter 3.). Schließlich sind Fälle zu erörtern, in denen die pünktliche Erbringung der Leistung dem Schuldner möglich, aber infolge der CoronaPandemie unzumutbar ist (unter 4.).

\section{Dauerhafte Unmöglichkeit der Leistungserbringung}

\section{Befreiung der Parteien von ihren wechselseitigen Leistungspflichten und Rücktrittsrecht des Gläubigers}

Kann der Schuldner infolge der Corona-Pandemie die vertraglich geschuldete Leistung dauerhaft nicht erbringen, wird er gem. $\$ 275$ Abs. 1 BGB von seiner Pflicht zur Leistung frei; er verliert aber zugleich gem. $\$ 326$ Abs. 1 BGB den Anspruch auf die Gegenleistung. Dies gilt unabhängig davon, ob die Unmöglichkeit bereits bei Vertragsschluss vorlag oder erst nachträglich eingetreten ist. Keine Rolle spielt auch, ob der geschlossene Vertrag auf einen einmaligen Leistungsaustausch gerichtet ist oder ob es sich um ein Dauerschuldverhältnis handelt.

$\mathrm{Zu}$ einer dauerhaften Unmöglichkeit infolge der Corona-Pandemie kommt es beispielsweise dann, wenn die Erbringung einer Leistung abgesagt werden muss und es sich dabei um ein absolutes Fixgeschäft handelt. 
Dies ist der Fall bei sämtlichen Leistungen, die im Zusammenhang mit der Durchführung zeitlich gebundener Massenveranstaltungen stehen, die infolge der Corona-Pandemie abgesagt werden. ${ }^{1}$ Kann der Gläubiger an einer solchen Veranstaltung nicht teilnehmen, hat er einen Anspruch auf Rückerstattung der Gegenleistung, d.h. des für eine Eintrittskarte gezahlten Kaufpreises. Gleiches gilt für Hotel- oder Flugbuchungen. Wurde die Zahlung geleistet, bevor der Anspruch des Schuldners auf die Gegenleistung erloschen ist, ergibt sich der Rückerstattungsanspruch aus $\$ 326$ Abs. 4 BGB; wurde geleistet, nachdem der Anspruch bereits erloschen war, ergibt er sich aus $\$ \mathbb{S} 812$ Abs. 1 Satz 1 Var. 1, 326 Abs. 1 BGB. ${ }^{2}$

Auch bei Dauerschuldverhältnissen wird der Gläubiger von der Gegenleistungspflicht frei, wenn der Schuldner die Leistung für einen bestimmten Zeitraum nicht erbringen kann. Kann der Gläubiger beispielsweise sein Fitnessstudio für eine bestimmte Zeit wegen einer behördlichen Schließungsanordnung nicht nutzen, muss er nach $\$ 326$ Abs. 1 BGB für diesen Zeitraum seine Mitgliedsbeiträge nicht entrichten. Dies gilt im Ergebnis selbst dann, wenn die Leistung theoretisch „nachgeholt“ werden könnte, indem die ausgefallene Zeit am Ende angehängt wird: Auch wenn es sich dann nicht um ein absolutes Fixgeschäft handeln sollte, ${ }^{3}$ wird jedenfalls der Gegenleistungsanspruch bis zur Nachholung der Leistung

1 MüKoBGB/W. Ernst, 8. Aufl. 2019, BGB $\$ 275$ Rn. 51; zur Einordnung der Durchführung eines Symphoniekonzerts als absolutes Fixgeschäft AG Dortmund, Urteil v. 13.10.1948 - 26 C 517/48, NJW 1949, 148; zum Kauf von Karten für ein Fußballspiel.

2 Wie hier MüKoBGB/W. Ernst, 8. Aufl. 2019, BGB $\$ 326$ Rn. 103. Die Differenzierung nach dem Zeitpunkt der Erbringung der Gegenleistung ergibt Sinn, weil es einen Unterschied macht, ob bestehende Leistungspflichten zunächst erfüllt und dann aufgrund von Leistungsstörungen rückabgewickelt werden - hierfür enthalten die $\int \mathbb{S} 346 \mathrm{ff}$. BGB ein vorrangiges und darauf zugeschnittenes Normenregime - oder ob auf eine tatsächlich nicht bestehende Schuld geleistet wird - das ist die klassische Bereicherungssituation. Zu undifferenziert erscheint daher die Regierungsbegründung zu $\ 326$ Abs. $4 \mathrm{BGB}$, wo es heißt, das Rücktrittsrecht sei generell besser auf die Rückabwicklung fehlgeschlagener Verträge zugeschnitten“, siehe BT-Drucks. 14/6040, S. 189. Für einen generellen Vorrang der Bereicherungshaftung dagegen $J$. Kobler, Bereicherungshaftung nach Rücktritt - eine verdrängte Verdrängung und ihre Folgen, AcP 208 (2008), S. 417 (443 ff.).

3 Vgl. BGH, Urteil v. 23.9.1992 - XII ZR 44/91, NJW 1992, 3226 (3228); MüKoBGB/ W. Ernst, 8. Aufl. 2019, BGB $₫ 275$ Rn. 52; BeckOK BGB/S. Lorenz, 53. Ed. 1.2.2020, BGB \275 Rn. 37; BeckOGK/T. Riehm, 1.2.2020, BGB \$275 Rn. 99; vgl. aber OLG Düsseldorf, Urteil v. 16.3.1989 - 10 U 142/88, BeckRS 1989, 05114, Rn. 60: Fixcharakter eines Dauerschuldverhältnisses (Raummiete), wenn der Beginn fest bestimmt ist. 
nicht durchsetzbar sein ( $\$ 320$ BGB). Daneben kann sich der Gläubiger von dem Dauerschuldverhältnis anlässlich eines auch nur vorübergehenden Leistungsausfalls auch vollständig lösen, indem er es nach $\$ 314$ BGB oder nach spezielleren Vorschriften wie etwa $\$ 626$ BGB außerordentlich kündigt. ${ }^{4}$ Wurde der Vertrag noch nicht in Vollzug gesetzt, besteht daneben ein Rücktrittsrecht nach $\$ 326$ Abs. 5 BGB. Wurde er bereits in Vollzug gesetzt, wird das Rücktrittsrecht durch das Kündigungsrecht verdrängt, da sonst die bereits ausgetauschten Leistungen nach Maßgabe der $\$ \$ 346$ ff. BGB zurück zu gewähren wären, was regelmäßig nicht im Interesse der Parteien liegt. ${ }^{5}$

Kann der Schuldner infolge der Corona-Pandemie die für eine Weiterverarbeitung oder eine Weiterveräußerung erforderlichen Rohstoffe nicht beziehen oder darf er kraft behördlicher Anordnung bestimmte Waren nur an bestimmte Abnehmer weiterveräußern, ${ }^{6}$ wird dies nur selten zur dauerhaften Unmöglichkeit der geschuldeten Leistung führen: Nämlich dann, wenn der Lieferengpass oder die behördliche Anordnung dauerhafter Natur ist (was kaum je der Fall sein dürfte) oder wenn es sich bei der Weiterveräußerung der ursprünglichen oder durch Weiterverarbeitung neu entstandenen Sache um ein absolutes Fixgeschäft handelt. Regelmäßig werden Unterbrechungen der Handelskette dagegen bloße Verzögerungen der Leistung (dazu unter 2.) oder allenfalls vorübergehende Unmöglichkeit (dazu unter 3.) zur Folge haben.

4 MüKoBGB/R. Gaier, 8. Aufl. 2019, BGB $₫ 314$ Rn. 18; BeckOGK/C. Herresthal, 1.6.2019, BGB $₫ 326$ Rn. 120; BeckOK BGB/S. Lorenz, 53. Ed. 1.2.2020, BGB $₫ 314$ Rn. 12.

5 Wie hier BeckOGK/C. Herresthal, 1.6.2019, BGB $\$ 326$ Rn. 120; Staudinger/R. Schwarze, 2015, Rn. B 56; a.A. MüKoBGB/W. Ernst, 8. Aufl. 2019, BGB \326 Rn. 109 (keine Verdrängung von $₫ 326$ Abs. 5 BGB durch $₫ 314$ BGB); BeckOK BGB/S. Lorenz, 53. Ed. 1.2.2020, BGB $\$ 314$ Rn. 13 (Vorrang des Unmöglichkeitsrechts).

6 Aus der Praxis wird berichtet, dass ein Chemikalienhandel Ethanol infolge der Corona-Pandemie ausschließlich für die Produktion von Desinfektionsmitteln verwenden oder an Hersteller von Desinfektionsmitteln weiterveräußern darf; ein Verkauf an andere Abnehmer ist behördlich untersagt. Siehe auch https:/www.faz. net/aktuell/wirtschaft/unternehmen/corona-herstellern-von-desinfektionsmitteln-g eht-ethanol-aus-16689993.html. 


\section{Schadensersatzpflicht des Schuldners}

Während die Befreiung der Parteien von ihren wechselseitigen Leistungspflichten von einem Vertretenmüssen des Schuldners unabhängig ist, setzen Schadensersatzansprüche des Gläubigers dies stets voraus. Dabei ist zwischen anfänglicher Unmöglichkeit ( $\$ 311$ a Abs. 2 BGB) und nachträglicher Unmöglichkeit ( $\mathbb{S} 280$ Abs. 1, Abs. 3, 283 BGB) zu differenzieren.

Ein Schadensersatzanspruch wegen anfänglicher Unmöglichkeit gem. $\$ 311$ a Abs. 2 BGB setzt voraus, dass der Schuldner das Leistungshindernis bei Vertragsschluss kannte oder kennen musste. Dem Schuldner wird hier nicht die anfängliche Unmöglichkeit als solche zum Vorwurf gemacht, sondern die Übernahme der Leistungspflicht wider besseres Wissen und die unterlassene Information des Gläubigers hierüber. ${ }^{7}$ Dies kommt in den hier interessierenden Fallkonstellationen nur dann in Betracht, wenn der Schuldner nicht nur Kenntnis von der Corona-Pandemie und den damit potentiell verbundenen Einschränkungen hatte, sondern darüber hinaus wusste oder hätte wissen müssen, dass ihm die Erfüllung des konkreten Vertrags nicht möglich sein werde. Solche Fälle dürften die Ausnahme sein. Zudem dürfte eine ähnliche Kenntnis dann regelmäßig auch auf Seiten des Gläubigers vorliegen, was zur Folge hat, dass ein etwaiger Schadensersatzanspruch jedenfalls gem. $\$ 254$ Abs. 1 BGB zu kürzen wäre. ${ }^{8}$

Tritt die Unmöglichkeit erst nach Vertragsschluss ein, ist Anknüpfungspunkt des Vertretenmüssens die Unmöglichkeit selbst. ${ }^{9}$ Beruht sie auf den Auswirkungen einer Pandemie, etwa hoheitlichen Anordnungen, kann dem Schuldners regelmäßig zumindest ein Verschuldensvorwurf nicht gemacht werden. ${ }^{10}$ Anderes gilt möglicherweise, wenn die Unmöglichkeit auf einer behördlichen Schließung des Betriebs des Schuldners beruht und den Schuldner daran eine Mitverantwortung triff, weil beispielsweise be-

7 Ausführlich zum Verhältnis der Haftung nach $\$ 311$ a Abs. 2 BGB zur c.i.c.-Haftung nach $\int \mathbb{S} 280$ Abs. 1, 241 Abs. 2, 311 Abs. 2 BGB MüKoBGB/W. Ernst, 8. Aufl. 2019, BGB $₫ 311$ a Rn. 21 f.; BeckOGK/C. Herresthal, 1.6.2019, BGB $₫ 311 \mathrm{a}$ Rn. 19 ff. (für Spezialität); J. Harke, Das neue Sachmängelrecht in rechtshistorischer Sicht, AcP 205 (2005), S. 67 (90 f.) (für Parallelität).

8 Für entsprechende Anwendung der Vorschrift MüKoBGB/W. Ernst, 8. Aufl. 2019, BGB $₫ 311$ a Rn. 68; BeckOGK/C. Herresthal, 1.6.2019, BGB $₫ 311$ a Rn. 129.

9 MüKoBGB/W. Ernst, 8. Aufl. 2019, BGB $\$ 283$ Rn. 5; BeckOGK/T. Riehm, 1.2.2020, BGB $\$ 283$ Rn. 28; abweichend BeckOK BGB/S. Lorenz, 53. Ed. 1.2.2020, BGB $₫ 283$ Rn. 3 (Umstände, aufgrund derer die Leistung unmöglich wurde).

10 Ebenso M.-P. Weller/M. Lieberknecht/V. Habrich, Virulente Leistungsstörungen Auswirkungen der Corona-Krise auf die Vertragsdurchführung, NJW 2020, 1017 (1019). 
triebliche Schutzvorkehrungen gegen eine Ausbreitung des Corona-Virus nicht getroffen wurden. Schadensersatzansprüche sind zudem denkbar in Fällen, in denen das Vertretenmüssen aufgrund der Übernahme einer Garantie oder des Beschaffungsrisikos zu bejahen ist.

\section{Verzögerung der Leistungserbringung}

Häufig wird die Corona-Pandemie aber nicht zu einer dauerhaften Unmöglichkeit, sondern lediglich zu einer Verzögerung der Leistung führen, etwa wenn sich Erfüllungshandlungen innerhalb der Handelskette aufgrund temporärer Produktionsstopps oder einer Überlastung von Speditionen verlangsamen.

\section{Zurückbehaltungsrecht und Rücktrittsrecht des Gläubigers}

Bei einer Verzögerung der Leistung gibt $₫ 320$ BGB dem nicht vorleistungsverpflichteten Gläubiger ein Zurückbehaltungsrecht hinsichtlich der Gegenleistung. Wird die Gegenleistung gleichwohl erbracht, kann sie allerdings nicht für die Dauer der Verzögerung der Leistung nach Bereicherungsrecht zurück gefordert werden: $\$ 813$ ermöglicht BGB die Rückforderung des trotz Bestehens einer Einrede Geleisteten nur bei peremtorischen Einreden. ${ }^{11}$

Zudem kann der Gläubiger dem Schuldner gem. $\$ 323$ Abs. 1 BGB eine angemessene Frist zur Leistung setzen und nach deren Ablauf vom Vertrag zurücktreten. Bei relativen Fixgeschäften kann der Rücktritt gem. $\$ 323$ Abs. 2 Nr. 2 BGB auch ohne vorherige Fristsetzung erfolgen. Wird ein Handelskauf als relatives Fixgeschäft abgeschlossen, lässt $\ 376$ Abs. 1 Satz 1 HGB einen Rücktritt unter erleichterten Voraussetzungen zu. Das Gesetz geht also davon aus, dass bei auch nur einseitigen Handelskäufen eine verspätete Erfüllung im Regelfall ausgeschlossen sein soll, ${ }^{12}$ weshalb der Gläu-

11 BGH, Urteil v. 22.4.1981 - VIII ZR 103/80, NJW 1982, 1587 (1588); OLG Zweibrücken, Urteil v. 19.2.2009 - 4 U 69/08, NJW 2009, 2221 (2222); MüKoBGB/M. Schwab, 7. Aufl. 2017, BGB $\$ 813$ Rn. 6; BeckOK BGB/C. Wendehorst, 53. Ed. 1.2.2020, BGB $₫ 813$ Rn. 4.

12 Kritisch zu dieser Annahme MüKoHGB/B. Grunewald, 4. Aufl. 2018, HGB $\$ 376$ Rn. 3; ausführlich zu den Unterschieden zwischen bürgerlich-rechtlichem und handelsrechtlichem Fixgeschäft und daraus resultierenden Wertungswidersprüchen C. Herresthal, Der Anwendungsbereich der Regelungen über den Fixhan- 
biger, der auf Erfüllung bestehen möchte, dies dem Schuldner sofort nach dem Ablauf der Zeit oder der Frist anzuzeigen hat ( $\$ 376$ Abs. 1 Satz 2 HGB).

\section{Schadensersatzpflicht des Schuldners}

Wie bei der Unmöglichkeit setzen Ansprüche des Gläubigers gegen den Schuldner auf Schadensersatz auch bei der Verzögerung der Leistung Vertretenmüssen voraus. Dies gilt sowohl für den Nichterfüllungsschaden ( $\mathbb{S} \int 280$ Abs. 1, Abs. 3, 281 BGB $)^{13}$ als auch für einen etwaigen Verzugsschaden ( $\$ \$ 280$ Abs. 1, Abs. 2, 286 BGB). Ein Verschulden des Schuldners wird sich bei für ihn unvorhersehbaren pandemiebedingten Verzögerungen nur ausnahmsweise begründen lassen: ${ }^{14}$ Etwa dann, wenn er es versäumt, absehbare Verzögerungen durch rechtzeitige Bevorratung zu verhindern oder bei Engpässen der „Hausspedition“ nach alternativen Transporteuren Ausschau zu halten. Ferner kann sich das Vertretenmüssen wiederum aus der Übernahme einer Garantie oder des Beschaffungsrisikos ergeben.

\section{Vorübergehende Unmöglichkeit der Leistungserbringung}

Gewissermaßen zwischen dauerhafter Unmöglichkeit und bloßer Verzögerung der Leistung ${ }^{15}$ liegen Fälle, in denen dem Schuldner die Leistung vorübergehend unmöglich ist - etwa wenn aufgrund der Auswirkungen der Corona-Pandemie bestimmte Waren für einen gewissen Zeitraum nicht lieferbar sind.

delskauf ( $\$ 376$ HGB) unter Berücksichtigung des reformierten Schuldrechts, ZIP 2006, S. 883.

13 Ob beim relativen Fixgeschäft die Fristsetzung im Rahmen von $₫ 281$ BGB entbehrlich ist, ist umstritten, da es an einer $₫ 323$ Abs. 2 Nr. 2 BGB vergleichbaren Regelung fehlt; für die Berücksichtigung des relativen Fixschuldcharakters im Rahmen der Interessenabwägung nach $₫ 218$ Abs. 2 BGB C. Behme, Übungsklausur Zivilrecht: „Pagode im Zweitlack“, JA 2019, S. 177 (179); M. Jaensch, Der Gleichlauf von Rücktritt und Schadensersatz, NJW 2003, S. 3613 (3615); BeckOK BGB/S. Lorenz, 53. Ed. 1.2.2020, BGB $\$ 281$ Rn. 30.

14 Vgl. auch M.-P. Weller/M. Lieberknecht/V. Habrich, Virulente Leistungsstörungen (Fn. 10), S. 1020.

15 Auf diese Zwischenstellung weist zutreffend A. Arnold, Die vorübergehende Unmöglichkeit nach der Schuldrechtsreform, JZ 2002, S. 866 (866) hin. 
Es ist absehbar, dass die Corona-Pandemie und die damit verbundenen Handelseinschränkungen - jedenfalls irgendwann - wieder enden. Fälle pandemiebedingter vorübergehender Unmöglichkeit werden daher häufiger sein als Fälle dauerhafter Unmöglichkeit. Nach der Rechtsprechung des BGH ist eine nur vorübergehende Unmöglichkeit der dauerhaften Unmöglichkeit jedoch gleichzustellen, wenn sie dazu führt, dass die Erreichung des Vertragszwecks in Frage gestellt ist und der einen oder anderen Partei bei billiger Abwägung der beiderseitigen Belange nicht mehr zugemutet werden kann, die Leistung dann noch zu fordern oder zu erbringen. ${ }^{16}$ Nicht vorübergehende, sondern dauerhafte Unmöglichkeit mit den daraus resultierenden Rechtsfolgen hat die Rechtsprechung auch angenommen, wenn die Leistungserbringung aufgrund von politischen Unruhen oder durch den Ausbruch eines Krieges am Erfüllungsort zwar nur für deren Dauer, also vorübergehend, unmöglich wird, aber das Ende dieser Unruhen für die Parteien nicht absehbar ist. ${ }^{17} \mathrm{Ob}$ die Unmöglichkeit auf politischen Unruhen oder den Auswirkungen einer Pandemie beruht, kann dabei keinen Unterschied machen. Je länger die Beeinträchtigungen des Wirtschaftslebens durch die Corona-Pandemie andauern und je weniger das Ende dieser Beeinträchtigungen absehbar ist, desto eher ist daher nicht von vorübergehender, sondern von dauerhafter Unmöglichkeit auszugehen.

\section{Befreiung der Parteien von ibren wechselseitigen Leistungspflichten und Rücktrittsrecht des Gläubigers}

Auch die vorübergehende Unmöglichkeit führt zu einer jedenfalls vorübergehenden Leistungsbefreiung des Schuldners nach $\$ 275$ Abs. 1 BGB; eine Klage gegen ihn ist als „zur Zeit unbegründet“ abzuweisen. ${ }^{18}$ Dementsprechend entfällt für die Dauer der Unmöglichkeit auch der Anspruch

16 BGH, Urteil v. 19.10.2007 - V ZR 211/06, NJW 2007, 3777 (3778f.); vgl. zu früheren Recht bereits BGH, Urteil v. 30.10.1953 - V ZR 76/52, BeckRS 1953, 31203262; BGH, Urteil v. 31.1.1967 - V ZR 125/65, NJW 1967, 721 (722); BGH, Urteil v. 11.3.1982 - VII ZR 357/80, NJW 1982, 1458 (1458).

17 BGH, Urteil v. 11.3.1982 - VII ZR 357/80, NJW 1982, 1458 (1458) m.w.N.

18 BGH, Urteil v. 16.9.2010 - IX ZR 121/09, NZI 2010, 956 (959); MüKoBGB/W. Ernst, 8. Aufl. 2019, BGB $₫ 275$ Rn. 141; BeckOK BGB/S. Lorenz, 53. Ed. 1.2.2020, BGB $₫ 275$ Rn. 39; BeckOGK/T. Riehm, 1.2.2020, BGB $₫ 283$ Rn. 28. 
auf die Gegenleistung nach $\$ 326$ Abs. 1 BGB. ${ }^{19}$ Konsequenterweise wird man dem Gläubiger auch das Recht zugestehen müssen, eine bereits erbrachte Gegenleistung zurückzuverlangen, und zwar ohne dass dafür eine Aufhebung des Vertrags erforderlich ist. ${ }^{20}$ Wiederum ergibt sich der Rückforderungsanspruch aus $\$ 326$ Abs. 4 BGB oder aus $\$ \mathbb{S} 812$ Abs. 1 Satz 1 Var. 1, 326 Abs. 1 BGB, je nachdem, ob die Gegenleistung erbracht wurde, bevor oder nachdem der Anspruch des Schuldners auf die Gegenleistung erloschen ist. ${ }^{21}$

Schon weil sich in Fällen vorübergehender Unmöglichkeit eine Schlechterstellung des Gläubigers gegenüber Fällen verbietet, in denen sich die Leistung lediglich verzögert, steht dem Gläubiger auch ein Rücktrittsrecht $\mathrm{zu}$, das sich nach herrschender Auffassung aus einer (mangels Fälligkeit der Leistung) analogen Anwendung des $\$ 323$ Abs. 1 BGB ergibt. ${ }^{22}$ Dabei ist freilich nicht recht ersichtlich, was mit der Anwendung von $\mathbb{3} 323$ Abs. 1 BGB anstelle des systematisch besser passenden $\$ 326$ Abs. 5 BGB gewonnen ist, zumal bei vorübergehender Unmöglichkeit dem Schuldner die Leistung innerhalb einer angemessenen Frist regelmäßig weiterhin nicht möglich sein wird, ohne dass der Schuldner dies beeinflussen könnte. Jedenfalls verliert das Fristsetzungserfordernis seinen Sinn, sobald sich die Aussicht, dass die Leistung wieder möglich wird, zerschlägt; das Rücktrittsrecht folgt dann ohne weiteres aus $\$ 326$ Abs. 5 BGB. ${ }^{23}$

\section{Schadensersatzpflicht des Schuldners}

Schadensersatzansprüche des Gläubigers können zum einen auf Ersatz des Verzögerungsschadens gerichtet sein. Dieser ist schon deswegen nach $\$ 280$

19 Wie hier C.-W. Canaris, Die Reform des Rechts der Leistungsstörungen JZ 2001, S. 499 (500); Staudinger/G. Caspers, 2019, BGB $\$ 275$ Rn. 50; a.A. BeckOGK/T. Riehm, 1.2.2020, BGB $\$ 275$ Rn. 164 (nur Einrede nach $\$ 320$ BGB); offen lassend MüKoBGB/W. Ernst, 8. Aufl. 2019, BGB \275 Rn. 144 (Anwendung von $\$ 326$ Abs. 1 BGB dürfte nicht erforderlich sein wegen ausreichenden Schutzes des Gläubigers durch $₫ 320$ BGB).

20 Wie hier C.-W. Canaris, Reform (Fn. 19), S. 500, der den Anspruch aus $\$ 326$ Abs. 4 BGB bejaht; a.A. A. Arnold, Vorübergehende Unmöglichkeit (Fn. 15), S. 868; MüKoBGB/W. Ernst, 8. Aufl. 2019, BGB $\$ 275$ Rn. 144; BeckOGK/T. Riehm, 1.2.2020, BGB $\$ 275$ Rn. 167.

21 Siehe oben unter II.1.b).

22 MüKoBGB/W. Ernst, 8. Aufl. 2019, BGB $\$ 275$ Rn. 151 f.; BeckOK BGB/S. Lorenz, 53. Ed. 1.2.2020, BGB $₫ 275$ Rn. 41.

23 MüKoBGB/W. Ernst, 8. Aufl. 2019, BGB \275 Rn. 146. 
Abs. 1 BGB ersatzfähig, ohne dass Verzug vorliegen müsste, weil Verzug eine fällige Leistung voraussetzen würde. ${ }^{24}$ Daneben kann der Gläubiger Schadensersatz statt der Leistung verlangen, der sich nach herrschender Auffassung aus $\mathbb{S} 280$ Abs. 1, Abs. 3, 281 BGB ergibt. ${ }^{25}$ Naheliegender erscheint es, die einschlägigen Anspruchsgrundlagen des Unmöglichkeitsrechts mit ihren unterschiedlichen Anknüpfungspunkten für das Vertretenmüssen heranzuziehen: Steht die vorübergehende Unmöglichkeit bereits bei Vertragsschluss fest, kommt daher ein Schadensersatzanspruch aus $\$ 311$ a Abs. 2 BGB in Betracht; dem Schuldner kann hier (nur) zum Vorwurf gemacht werden, dass er sich wider besseres Wissen auf die vertragliche Leistungspflicht eingelassen hat, obwohl er die vorübergehende Unmöglichkeit kannte oder kennen musste. Ist die pandemiebedingte vorübergehende Unmöglichkeit erst nach Vertragsschluss eingetreten, resultiert der Schadensersatzanspruch aus $\$ \mathbb{S} 280$ Abs. 1, Abs. 3, 283 BGB $^{26}$ und der Anknüpfungspunkt für ein etwaiges Vertretenmüssen ist die Unmöglichkeit selbst; wie bereits beim Rücktrittsrecht erscheint auch hier das in $\$ 281$ BGB statuierte Fristsetzungserfordernis nicht sinnvoll.

\section{Unzumutbarkeit der Leistungserbringung}

Schließlich sind Fälle denkbar, in denen dem Schuldner die Erbringung der objektiv noch möglichen Leistung infolge der Corona-Pandemie unzumutbar wird, mit der Konsequenz, dass der Schuldner die Leistung gem. $\$ 275$ Abs. 2 oder Abs. 3 BGB verweigern kann.

Allerdings werden sich die engen Voraussetzungen eines Leistungsverweigerungsrechts nach $\$ 275$ Abs. 2 oder Abs. 3 BGB mit den Auswirkungen der Corona-Pandemie nur selten begründen lassen. ${ }^{27}$ Eine in diesem

24 Wie hier MüKoBGB/W. Ernst, 8. Aufl. 2019, BGB $₫ 275$ Rn. 153; BeckOK BGB/S. Lorenz, 53. Ed. 1.2.2020, BGB $\$ 275$ Rn. 68; a.A. BeckOGK/T. Riehm, 1.2.2020, BGB $₫ 275$ Rn. 168; offen lassend BGH, Urteil v. 12.3.2013 - XI ZR 227/12, NJW 2013, 3437 (3439).

25 MüKoBGB/W. Ernst, 8. Aufl. 2019, BGB \275 Rn. 155; BeckOK BGB/S. Lorenz, 53. Ed. 1.2.2020, BGB $₫ 275$ Rn. 68; BeckOGK/T. Riehm, 1.2.2020, BGB $₫ 275$ Rn. 160.

26 Ebenso C.-W. Canaris, Reform (Fn. 19), S. 500.

27 Für eine großzügige Handhabung von $₫ 275$ Abs. 2 BGB M.-P. Weller/M. Lieberknecht/V. Habrich, Virulente Leistungsstörungen (Fn. 10), S. 1020: In der „Weltviruskrise" soll das Leistungsinteresse des Gläubigers niedriger anzusetzen sein, soweit er ohnehin außerstande wäre, den geschuldeten Gegenstand wie beabsichtigt zu verwenden. 
Zusammenhang bislang, soweit ersichtlich, nicht diskutierte Problematik betrifft Fälle, in denen eine Leistung nicht aus rechtlichen Gründen unmöglich wird, sondern lediglich von behördlicher Seite empfohlen wird, die Leistung nicht zu erbringen bzw. nicht in Anspruch zu nehmen. So ist beispielsweise denkbar, dass öffentliche Veranstaltungen in absehbarer Zeit wieder erlaubt werden, Behörden oder Gesundheitsexperten aber empfehlen, von solchen Veranstaltungen weiterhin Abstand zu nehmen. Darf die Opernsängerin einen erlaubten Auftritt mit der Begründung absagen, sie habe nach wie vor Sorge vor dem Infektionsrisiko und möchte sich daher von öffentlichen Veranstaltungen (natürlich unter Verzicht auf ihre Gage) lieber fernhalten? Die Frage wird sich nicht pauschal bejahen oder verneinen lassen. ${ }^{28}$ Allerdings müssen schon besondere Umstände des Einzelfalles vorliegen, die dazu führen, dass in einer solchen Situation die im Rahmen des $₫ 275$ Abs. 3 BGB gebotene Abwägung des persönlichen Leistungshindernisses des Schuldners mit dem Leistungsinteresse des Gläubigers zugunsten des Schuldners ausfällt. Dies kann etwa dann der Fall sein, wenn der Schuldner aufgrund von Vorerkrankungen oder seines Alters zu einer besonderen Risikogruppe zählt.

\section{Zwischenfazit: Gesetzliche Zuweisung des Risikos pandemiebedingter Leistungsstörungen an den Schuldner}

Mit gewissen Unterschieden hinsichtlich der konkreten Voraussetzungen und der dogmatischen Herleitung hat der Gläubiger bei allen in Folge der Corina-Pandemie möglicherweise auftretenden Leistungsstörungen das Recht, die Gegenleistung zu verweigern und sich vom Vertrag zu lösen. Schadensersatzansprüche werden nur ausnahmsweise in Betracht kommen, da sie das Vertretenmüssen des Schuldners voraussetzen, das sich bei pandemiebedingten Leistungsstörungen vor allem bei Übernahme einer Garantie oder eines Beschaffungsrisikos bejahen lässt.

Alle hier skizzierten Fälle von Leistungsstörungen haben gemeinsam, dass innerhalb der jeweiligen vertraglichen Beziehung das Risiko der Leistungsstörung der Schuldner trägt: Er ist es, der den vertraglichen Anspruch auf die Gegenleistung spätestens dann verliert, wenn der Gläubiger vom

28 Für eine großzügige Handhabung von $₫ 275$ Abs. 3 BGB M.-P. Weller/M. Lieberknecht/V. Habrich, Virulente Leistungsstörungen (Fn. 10), S. 1020 mit Blick auf die Absage von Veranstaltungen (Rücksichtspflicht gem. $₫ 241$ Abs. 2 BGB gegenüber der Gesamtheit der Besucher). 
Vertrag wirksam zurückgetreten ist, und zwar unabhängig davon, ob er die Leistungsstörung zu vertreten hat oder nicht. Dass ihn an einer pandemiebedingten Unmöglichkeit oder Verzögerung der Leistung ebenso wenig die Schuld trifft wie den Gläubiger, hilft ihm nicht, sondern bewahrt ihn lediglich vor über den Verlust der Vergütung hinausgehenden Schadensersatzansprüchen.

In Lieferketten führt die geltende Rechtslage dazu, dass eine Leistungsstörung am Beginn der Lieferkette, die in den nachgelagerten Vertragsbeziehungen ebenfalls zu Leistungsstörungen führt, zur Folge hat, dass eine Reihe von Unternehmen als Gläubiger zwar die ausfallenden Leistungen ihrer Lieferanten nicht bezahlen muss, gleichzeitig als Schuldner aber auch keine Vergütung von ihren Kunden erhält. Am Ende der Lieferkette steht eine Vielzahl von Gläubigern (häufig Verbrauchern), die keine Leistung erhalten, aber auch keine Gegenleistung erbringen müssen bzw. eine bereits erbrachte Gegenleistung zurück verlangen können.

Dagegen ist das Risiko, dass innerhalb einer vertraglichen Beziehung eine Leistung erbracht wird, die der Gläubiger - etwa aufgrund einer vorübergehenden Schließung seines Betriebs - nicht verwenden kann, dem Gläubiger zugewiesen. Nur in Ausnahmefällen kommt ein Anspruch des Gläubigers auf Vertragsanpassung oder ein Rücktrittsrecht wegen einer Störung der Geschäftsgrundlage nach $\$ 313$ BGB in Betracht. Die Geschäftsgrundlagenstörung kann nämlich nur den Inhalt eines konkreten Vertrags, nicht aber die vertragliche oder gesetzliche Risikoverteilung als solche ändern oder korrigieren. ${ }^{29} \$ 313$ Abs. 1 BGB betont deshalb ausdrücklich, dass die Zumutbarkeit des Festhaltens am unveränderten Vertrag unter Berücksichtigung der Risikoverteilung zu beurteilen ist, die sich aus dem Vertrag oder aus dem Gesetz ergibt.

Diese Risikoverteilung ist unter „normalen“ Umständen ohne weiteres sachgerecht: Leistungsstörungen haben ihre Ursache in der Sphäre des Schuldners, sie sind für den Schuldner beherrschbar und vermeidbar - zumindest eher beherrschbar und vermeidbar als für den Gläubiger, der sie überhaupt nicht beeinflussen kann. So kann beispielsweise der Verkäufer einer Ware, die von seinem Vorlieferanten nicht ausgeliefert wird, seine Bezugsquelle wechseln; er kann eine defekte Maschine, die zu einem Produktionsausfall und in der Folge zu einer Lieferverzögerung führt, reparieren oder auswechseln, oder er kann sich gegen derartige Szenarien versichern. Folgerichtig weist das Gesetz dem Gläubiger das Risiko der Leistungsstörung nur ganz ausnahmsweise zu - nämlich wenn er für die Leis- 
tungsstörung allein oder weit überwiegend verantwortlich ist oder wenn sie zu einer Zeit eintritt, zu welcher er sich in Annahmeverzug befindet. In diesem Fällen muss der Gläubiger trotz Unmöglichkeit die Gegenleistung erbringen ( $\$ 326$ Abs. 2 Satz $1 \mathrm{BGB})$; sein Rücktrittsrecht ist ausgeschlossen ( $\$ 323$ Abs. 6 BGB).

\section{B. Auswirkungen der gesetzlichen Risikoverteilung}

Die gesetzliche Risikoverteilung soll im Folgenden an drei Fallbeispielen verdeutlicht werden. Fallbeispiel 1 betrifft eine klassische Lieferkette, Fallbeispiel 2 eine ausgefallene Konzertveranstaltung, Fallbeispiel 3 die Lieferung einer mangelhaften Sache.

\section{Fallbeispiel 1 - Lieferkette}

A ist Lieferant von Holz. Er verkauft dieses an den Hersteller B. Dieser benötigt das Holz, um daraus Kleinmöbel herzustellen, die er an die Großhändler C1 und C2 verkauft. Diese beliefern damit die Einzelhändler D1 D10, die schließlich an die Endabnehmer (Verbraucher) E1 bis E1000 weiterverkaufen. Aus Vereinfachungsgründen soll dabei unterstellt werden, dass die Unternehmen auf allen Stufen der Handelskette (A bis D) Aufwendungen in Höhe von 8.000 Währungseinheiten (WE) zu tragen haben und einen Gewinn von 2.000 WE erwirtschaften.

\begin{tabular}{|l|l|l|l|l|}
\hline & Einkaufspreis & $\begin{array}{l}\text { Eigene Aufwendun- } \\
\text { gen }\end{array}$ & Veräußerungspreis & $\begin{array}{l}\text { Gewinn } \\
\text { Verlust }\end{array}$ \\
\hline A & 0 & $8.000 \mathrm{WE}$ & $10.000 \mathrm{WE}$ & $+2.000 \mathrm{WE}$ \\
\hline B & $10.000 \mathrm{WE}$ & $8.000 \mathrm{WE}$ & $20.000 \mathrm{WE}$ & $+2.000 \mathrm{WE}$ \\
\hline $\begin{array}{l}\text { C1 und } \\
\text { C2 }\end{array}$ & $2 \times 10.000 \mathrm{WE}$ & $8.000 \mathrm{WE}$ & $30.000 \mathrm{WE}$ & $+2.000 \mathrm{WE}$ \\
\hline $\begin{array}{l}\text { D1 bis } \\
\text { D10 }\end{array}$ & $10 \times 3.000 \mathrm{WE}$ & $8.000 \mathrm{WE}$ & $40.000 \mathrm{WE}$ & $+2.000 \mathrm{WE}$ \\
\hline $\begin{array}{l}\text { E1 bis } \\
\text { E1.000 }\end{array}$ & $1.000 \times 40 \mathrm{WE}$ & $0 \mathrm{WE}$ & $0 \mathrm{WE}$ & $0 \mathrm{WE}$ \\
\hline
\end{tabular}

Addiert man die Gewinne der Unternehmen A, B, C1, C2 sowie D1 bis D10, ergibt sich in der Handelskette ein Gesamtgewinn von 8.000 WE.

Im Folgenden sei unterstellt, dass B infolge der Corona-Pandemie seinen Betrieb schließen muss. Gleichwohl muss er, da ihn das Risiko der Verwertbarkeit der Leistung des A triff, diese weiterhin bezahlen: A ist 
nach wie vor in der Lage, das bestellte Holz an B zu liefern; eine Leistungsstörung im Verhältnis zwischen A und B liegt nicht vor. Ferner wird unterstellt, dass die Voraussetzungen für einen Rücktritt des B vom Vertrag mit A nach $₫ 313$ Abs. 3 BGB nicht vorliegen. Kann B das Holz nicht weiterverarbeiten, entsteht kein Produkt, dass er an $\mathrm{C} 1$ und $\mathrm{C} 2$ weiter veräußern könnte. Er wird von seiner Pflicht zur Leistung gegenüber $\mathrm{C} 1$ und $\mathrm{C} 2$ gem. $\ 275$ Abs. 1 BGB frei und verliert gem. $\$ 326$ Abs. 1 BGB den Anspruch auf die Gegenleistung. Erhalten C1 und C2 von B keine Ware, können sie diese nicht an D1 bis D10 weiterveräußern; sie werden seinerseits von ihrer Pflicht zur Leistung gegenüber D1 bis D10 gem. $\mathbb{} 275$ Abs. 1 BGB frei und verlieren den Anspruch auf die Gegenleistung. Erhalten die Einzelhändler D1 bis D10 von C1 und C2 keine Ware, können sie schließlich ihre bestehenden Verträge mit den Kunden E1 bis E1000 nicht erfüllen (bzw. von vornherein keine Verträge mit Endkunden über den Kauf von Kleinmöbeln schließen). Sie verzeichnen daher ebenfalls keinerlei Einnahmen. Ferner soll - wiederum vereinfachend - unterstellt werden, dass sich die Aufwendungen aller Unternehmen in der Handelskette, die von ihrer Pflicht zur Leistung frei werden, halbieren.

Daraus ergibt sich das folgende Bild:

\begin{tabular}{|l|l|l|l|l|}
\hline & Einkaufspreis & $\begin{array}{l}\text { Eigene Aufwendun- } \\
\text { gen }\end{array}$ & Veräußerungspreis & $\begin{array}{l}\text { Gewinn / } \\
\text { Verlust }\end{array}$ \\
\hline A & $0 \mathrm{WE}$ & $8.000 \mathrm{WE}$ & $10.000 \mathrm{WE}$ & $+2.000 \mathrm{WE}$ \\
\hline B & $10.000 \mathrm{WE}$ & $4.000 \mathrm{WE}$ & $0 \mathrm{WE}$ & -14.000 \\
& & $4.000 \mathrm{WE}$ & $0 \mathrm{WE}$ \\
\hline $\begin{array}{l}\text { C1 und } \\
\text { C2 }\end{array}$ & 0 WE & $4.000 \mathrm{WE}$ & $0 \mathrm{WE}$ & $-4.000 \mathrm{WE}$ \\
\hline $\begin{array}{l}\text { D1 bis } \\
\text { D10 }\end{array}$ & 0 WE & 0 WE & 0 WE & $-4.000 \mathrm{WE}$ \\
\hline $\begin{array}{l}\text { E1 bis } \\
\text { E1.000 }\end{array}$ & 0 WE & & & $0 \mathrm{WE}$ \\
\hline
\end{tabular}

Addiert man die Gewinne und Verluste der Unternehmen A, B, C1 und C2 sowie D1 bis D10, ergibt sich in der Handelskette ein Gesamtverlust von -20.000 WE.

Die Ursache der Leistungsstörungen - hier also auf allen Ebenen die Auswirkungen der Corona-Pandemie - spielt dabei keine Rolle. 


\section{Fallbeispiel 2: Abgesagtes Konzert}

$\mathrm{X}$ ist Veranstalter von Konzerten. Für die Durchführung eines Konzerts mietet er eine Halle bei Vermieter $\mathrm{V}$ und engagiert die berühmte Pianistin P. Er verkauft Karten an die Konzertbesucher K1 bis K1000. Es sei aus Gründen der Vereinfachung unterstellt, dass alle Beteiligten (X, V und P) mit dem Konzert einen Gewinn von 10.000 WE erwirtschaften. V hat Aufwendungen (Bewirtschaftung der Halle) in Höhe von 20.000 WE zu tragen, die von ihm vereinnahmte Miete beträgt 30.000 WE. P hat keine Aufwendungen. X muss $\mathrm{V}$ und $\mathrm{P}$ bezahlen und hat zusätzlich interne Aufwendungen i.H.v. 10.000 WE; er verkauft die Karten an K1 bis K1.000 für jeweils $60 \mathrm{WE}$.

\begin{tabular}{|l|l|l|l|}
\hline & Eigene Aufwendungen & Vergütung & Gewinn / Verlust \\
\hline Vermieter V & $20.000 \mathrm{WE}$ & $30.000 \mathrm{WE}$ & $+10.000 \mathrm{WE}$ \\
\hline Pianistin P & $0 \mathrm{WE}$ & $10.000 \mathrm{WE}$ & $+10.000 \mathrm{WE}$ \\
\hline Veranstalter X & $\begin{array}{l}40.000 \mathrm{WE} \text { extern }+10.000 \mathrm{WE} \\
\text { intern }\end{array}$ & $60.000 \mathrm{WE}$ & $+10.000 \mathrm{WE}$ \\
\hline K1 bis K1.000 & $1.000 \times 60 \mathrm{WE}$ & $0 \mathrm{WE}$ & $0 \mathrm{WE}$ \\
\hline
\end{tabular}

Addiert man die Gewinne der Unternehmer V, P und X, ergibt sich ein Gesamtgewinn von $30.000 \mathrm{WE}$.

Im Folgenden sei unterstellt, dass das Konzert infolge der Corona-Pandemie nicht stattfinden kann. Ferner wird unterstellt, dass $\mathrm{X}$ von dem Mietvertrag mit V deswegen gem. $₫ 313$ Abs. 3 BGB zurücktreten kann. Ferner kann $\mathrm{P}$ ihre Leistung nicht erbringen und wird gem. $\mathbb{2 7 5}$ Abs. 1 $\mathrm{BGB}$ von ihrer Leistungspflicht frei, erhält aber von X deswegen gem. $\$ 326$ Abs. 1 BGB auch keine Vergütung. Findet das Konzert nicht statt, kann X seine Verträge mit den Konzertbesuchern K1 bis K.1000 nicht erfüllen; er wird (weil es sich bei dem Konzert um ein absolutes Fixgeschäft handelt) daher gem. $\$ 275$ Abs. 1 BGB von seiner Pflicht zur Leistung frei und erhält gem. $\$ 326$ Abs. 1 BGB keine Gegenleistung. Erneut soll - wiederum vereinfachend - unterstellt werden, dass sich die Aufwendungen aller Unternehmer, die von ihrer Pflicht zur Leistung frei werden, halbieren.

Daraus ergibt sich das folgende Bild:

\begin{tabular}{|l|l|l|l|} 
& \multicolumn{1}{|c|}{ Eigene Aufwendungen } & \multicolumn{1}{c|}{ Vergütung } & \multicolumn{1}{c|}{$\begin{array}{c}\text { Gewinn / Ver- } \\
\text { lust }\end{array}$} \\
\hline Vermieter V & $10.000 \mathrm{WE}$ & $0 \mathrm{WE}$ & $-10.000 \mathrm{WE}$ \\
\hline Pianistin P & $0 \mathrm{WE}$ & $0 \mathrm{WE}$ & $0 \mathrm{WE}$ \\
\hline Veranstalter X & $5.000 \mathrm{WE}$ (intern) & $0 \mathrm{WE}$ & $-5.000 \mathrm{WE}$
\end{tabular}




\begin{tabular}{|l|l|l|l|}
\hline & \multicolumn{1}{|c|}{ Eigene Aufwendungen } & Vergütung & $\begin{array}{c}\text { Gewinn / Ver- } \\
\text { lust }\end{array}$ \\
\hline K1 bis K1.000 & 0 WE & 0 WE & $0 \mathrm{WE}$ \\
\hline
\end{tabular}

Addiert man die Verluste der Unternehmer V, P und X, ergibt sich ein Gesamtverlust von -15.000 WE.

\section{Fallbeispiel 3: Lieferung einer mangelhaften Sache}

K erwirbt bei Autohändler V einen Sportwagen. Einen Tag nach Lieferung fällt das Navigationssystem permanent aus. Welche Rechte hat K?

Dieser an sich geradezu banale Fall wird hier nur deshalb erwähnt, um zu illustrieren, dass es sich bei der Schlechtleistung um eine Leistungsstörung handelt, die von der Corona-Pandemie grundsätzlich völlig unabhängig ist; weder wird sie durch die Corona-Pandemie verursacht noch ändert sich deswegen ihre rechtliche Bewertung. In Einzelfällen ist allerdings denkbar, dass die Frage der Behebbarkeit eines Mangels aufgrund der Auswirkungen der Corona-Pandemie anders zu beurteilen ist, etwa wenn die Nacherfüllung aufgrund von Betriebsschließungen im Kontext der Corona-Pandemie (vorübergehend) unmöglich wird. In diesen Fällen muss, obwohl der Mangel an sich behebbar wäre, das Erfordernis der Fristsetzung für die Ausübung fristgebundener Gewährleistungsrechte (Rücktritt, Minderung, Schadensersatz statt der Leistung) entfallen; das Rücktrittsrecht des Käufers folgt also aus $₫ 437$ Nr. 2 i.V.m. $\$ 326$ Abs. 5 BGB (statt i.V.m. $\$ 323$ Abs. $1 \mathrm{BGB}$ ) und ein etwaiger Anspruch auf Schadensersatz statt der Leistung aus $₫ 437$ Nr.3 i.V.m. $\$ 311$ a Abs. 2 BGB bzw. $\mathbb{S} 280$ Abs. 1, Abs. 3, 283 BGB (statt i.V.m. $\$ \mathbb{S} 280$ Abs. 1, Abs. 3, 281 BGB). Ein an sich behebbarer Mangel ist dann also zu behandeln wie ein unbehebbarer Mangel.

\section{Zwischenfazit und rechtsökonomische Bewertung}

Sowohl in Fall 1 als auch in Fall 2 erleiden die betroffenen Unternehmer in ihrer Eigenschaft als Schuldner erhebliche Verluste. Das Problem besteht dabei nicht darin, dass sie die Gegenleistung für die versprochene Leistung nicht erhalten - immerhin müssen sie auch die Leistung nicht erbringen. Am Beispiel des Herstellers B in Fallbeispiel 1 illustriert: Er kann und muss die Leistung gegenüber $\mathrm{C} 1$ und $\mathrm{C} 2$ nicht erbringen, erhält deswegen von ihnen auch keine Gegenleistung, behält aber zumindest das 
von A erhaltene Holz und erspart (zunächst) den mit der Weiterverarbeitung verbundenen Aufwand. Bilanziell verschlechtert sich seine Situation daher nur unwesentlich; auf der Aktivseite der Bilanz stehen Rohstoffe anstelle des Geldes, das er von C1 und C2 erhalten hätte. Eine solche Sichtweise greift aber zu kurz: B erspart nämlich nur einen Teil des mit der Weiterverarbeitung des Rohstoffs verbundenen Aufwandes (im Beispiel wurde mit $50 \%$ Ersparnis kalkuliert, was aber je nach den Umständen des Einzelfalls noch deutlich weniger sein kann). Ein großer Teil der Fixkosten läuft weiter, auch wenn B nicht produziert. Dies dürfte für den Großteil aller Unternehmen, die aufgrund der Corona-Pandemie ihre Leistungen nicht erbringen und dementsprechend keine Vergütung vereinnahmen können, in ähnlicher Weise gelten. Ihnen drohen erhebliche Liquiditätsengpässe, die auf absehbare Zeit zur Zahlungsunfähigkeit und damit zur Insolvenz führen können.

Auf der anderen Seite stehen am Ende der jeweiligen Leistungsketten zahlreiche Gläubiger, die zwar einerseits nicht in den Genuss der bestellten Leistung (Ware oder Konzertbesuch) gelangen, die aber andererseits jedenfalls liquiditätsmäßig auch nicht belastet werden, weil sie von ihrer Gegenleistungspflicht bereits kraft Gesetzes nach $\$ 326$ Abs. 1 BGB frei werden oder jedenfalls die Gegenleistung zurück verlangen und vom Vertrag zurücktreten können.

Unter normalen Umständen ist diese Risikoverteilung sachgerecht, weil Leistungsstörungen typischerweise der Sphäre des Schuldners zuzuordnen sind. Sie entspricht daher auch dem, was unter normalen Umständen die Parteien vereinbaren würden, wenn sie das Risiko einer Leistungsstörung vertraglich einer Partei zuweisen würden. Die Regelungen des dispositiven bürgerlichen Leistungsstörungsrechts werden insofern dem Anspruch an das dispositive Recht, möglichst den hypothetischen Parteiwillen abzubilden und auf diese Weise den Parteien den Abschluss unvollständiger Verträge zu ermöglichen, ${ }^{30}$ gerecht.

In Situationen wie der Corona-Pandemie ist dies grundlegend anders: Das Risiko einer Pandemie ist nicht der Sphäre des Schuldners zuzuordnen, weil es alle Unternehmen weltweit betrifft und von keiner Vertragspartei eher "beherrscht“ werden kann als von der anderen Vertragspartei. Darin liegt ein wesentlicher Unterschied zu einer regional begrenzten Epidemie: Sie ist der Pandemie nur dann vergleichbar, wenn beide Vertrags-

30 Zur Entlastungsfunktion des dispositiven Rechts H.-B. Schäfer/C. Ott, Lehrbuch der ökonomischen Analyse des Zivilrechts, 5. Aufl., Berlin / Heidelberg 2012, S. $455 \mathrm{f}$. 
parteien von ihr betroffen sind; ist ausschließlich der Schuldner von der Epidemie betroffen, fällt sie ebenso in seine Risikosphäre wie eine lokal auftretende Unwetterlage.

Bei einer weltweiten Pandemie entspricht es nicht dem hypothetischen Willen der Parteien, dass stets der Schuldner das Risiko von Leistungsstörungen, die von einer solchen Pandemie verursacht werden, allein trägt. Hätten die Parteien das Auftreten einer solchen Pandemie vorhergesehen, hätten sie das Risiko vertraglich vermutlich geteilt (etwa durch eine teilweise Aufrechterhaltung des vertraglichen Vergütungsanspruchs oder die Knüpfung eines Rücktritts an zusätzliche, über $₫ 323$ Abs. 1 BGB hinausgehende Voraussetzungen). Denkbar ist auch, dass die Parteien das Risiko vertraglich einer der Parteien allein zugewiesen hätten; eine solche Risikoübernahme wäre dann aber vergütet worden: Der Schuldner hätte eine höhere Vergütung seiner Leistung für die Risikoübernahme verlangt, der Gläubiger einen Rabatt auf die Leistung.

\section{Alternativvorschlag: Zuweisung des Risikos pandemiebedingter Leistungsstörungen an den Gläubiger}

Welche Art der Risikoteilung oder Risikozuweisung die Parteien anstelle der einseitigen gesetzlichen Risikozuweisung an den Schuldner vereinbart hätten, hängt stark von den Umständen des Einzelfalles ab und lässt sich nicht pauschal beantworten. Daher ist es dem Gesetzgeber nicht möglich, durch dispositives Leistungsstörungsrecht den hypothetischen Willen der Parteien mit Blick auf die Behandlung pandemiebedingter Leistungsstörungen abzubilden. Das Gesetz kann hier nur mit Schwarz/Weiß-Lösungen arbeiten und die Parteien, sofern sie eine Lösung innerhalb des unendlich weiten Graubereichs wünschen, auf vertragliche Gestaltungen verweisen.

Im Folgenden soll gezeigt werden, dass der Gesetzgeber gleichwohl eine Lösung wählen könnte, die rechtsökonomisch überzeugender wäre als die Zuweisung des Risikos pandemiebedingter Leistungsstörungen an den Schuldner: nämlich das genaue Gegenteil, eine Zuweisung des Risikos pandemiebedingter Leistungsstörungen an den Gläubiger.

Die Zuweisung des Risikos pandemiebedingter Leistungsstörungen an den Gläubiger ließe sich rechtstechnisch einfach umsetzen. Das Gesetz kennt bereits Fälle, in denen ausnahmsweise der Gläubiger die Preisgefahr trägt, wenn dem Schuldner die Leistung unmöglich ist, nämlich wenn er für den Umstand, aufgrund dessen der Schuldner nach $\$ 275$ Abs. 1 bis 3 nicht zu leisten braucht, weit überwiegend verantwortlich ist, oder wenn 
dieser vom Schuldner nicht zu vertretende Umstand zu einer Zeit eintritt, zu welcher der Gläubiger im Verzug der Annahme ist ( $\$ 326$ Abs. 2 Satz 1 BGB). Unter denselben Voraussetzungen lässt $\$ 323$ Abs. 6 BGB das Rücktrittsrecht des Schuldners entfallen. Diese Vorschriften könnten um die Situation einer Pandemie - oder allgemeiner gesprochen: um die Situation eines Ereignisses, von dem beide Parteien gleichermaßen betroffen sind und das von beiden Parteien gleichermaßen nicht beherrschbar ist - erweitert werden.

Der Effekt wäre, dass in einer von der Corona-Pandemie betroffenen Leistungsbeziehung die jeweiligen Schuldner ihre Vergütungsansprüche behielten. Gläubiger könnten sich nicht vom Vertrag lösen und eine bereits erbrachte Gegenleistung nicht zurückverlangen. Im (seltenen) Falle einer dauerhaften Unmöglichkeit der Leistungserbringung würden sie somit für eine Leistung bezahlen, die sie tatsächlich nicht erhalten. In den (weitaus häufigeren) Fällen einer vorübergehenden Unmöglichkeit oder einer bloßen Verzögerung der Leistung müssten sie auf die Leistung warten. In der Leistungskette würde der pandemiebedingte Schaden somit einerseits weit nach hinten verlagert und auf der anderen Seite auf eine Vielzahl von Gläubigern verteilt (loss spreading) ${ }^{31}$. Diese könnten den auf sie entfallenden summenmäßig geringen Schaden leichter tragen als der Schuldner, der nicht nur in einer Vertragsbeziehung von seinem Gläubiger, sondern von einer Vielzahl von Gläubigern in einer Vielzahl von Vertragsbeziehungen keine Vergütung erhält und erst durch diese Kumulierung erloschener Ansprüche in Liquiditätsprobleme gerät.

Der hiesige Vorschlag würde somit die Gefahr von Liquiditätsengpässen zumindest in den Fällen abwenden, in denen der Gläubiger die Leistung bereits bezahlt hat und sie infolge einer derartigen Regelung nicht zurückverlangen könnte. In Fällen, in denen der Gläubiger die Gegenleistung noch nicht erbracht hat, könnte er sie weiterhin nach $\$ 320$ BGB bis zur Bewirkung der Leistung verweigern, es sei denn, er ist vorleistungspflichtig. Anders gewendet: Mit der Vorleistung würde er nicht mehr nur - wie bisher - das Insolvenzrisiko des Schuldners auf sich nehmen, sondern auch das Pandemierisiko. Ausnahmesituationen, in denen den Gläubiger die Übernahme dieses Risikos wirtschaftlich überfordert, könnten ihrerseits durch eine Härtefallregelung in Gestalt einer Rückausnahme zu $\$ 326$ Abs. 2 BGB und $\$ 323$ Abs. 6 BGB abgefedert werden.

31 Grundlegend G. Calabresi, The Costs of accidents - a legal and economic analysis, 1970, S. $39 \mathrm{ff}$. 


\section{Auswirkungen auf Fallbeispiel 1}

In Fallbeispiel 1 würde die gesetzliche Zuweisung des Risikos pandemiebedingter Leistungsstörungen an den Gläubiger dazu führen, dass B weiterhin gegenüber $\mathrm{C} 1$ und $\mathrm{C} 2$ von seiner Leistungspflicht gem. $\ 275$ Abs. 1 BGB frei würde. Er behielte jedoch gem. $\$ 326$ Abs. 2 BGB den Anspruch auf die Gegenleistung, der um die ersparten Aufwendungen i.H.v. 4.000 gekürzt wurde. Gleiches würde für die Leistungsbeziehung zwischen C1 / C2 und D1 bis D10 gelten. Diese schließlich würden gem. $\$ 275$ Abs. 1 BGB von ihrer Pflicht zur Leistung gegenüber E1 bis E1.000 frei, behielten aber ebenfalls ihre Ansprüche auf die Gegenleistung. Die Anrechnung der ersparten Aufwendungen auf allen Ebenen der Handelskette würde dazu führen, dass E1 bis E1.000 für die nicht erhaltene Ware 28 WE (statt ursprünglich $40 \mathrm{WE}$ ) bezahlen müssten. Wurde eine darüber hinausgehende Gegenleistung erbracht, kann der darüber hinausgehende Betrag gem. $\$ 326$ Abs. 4 BGB zurückverlangt werden. In Fällen bloß vorübergehender Unmöglichkeit würde der Leistungsanspruch wieder aufleben, sobald die Leistung wieder möglich wird. In diesem Falle würde auch der Gegenleistungsanspruch wieder in voller Höhe aufleben.

\begin{tabular}{|c|c|c|c|c|}
\hline & Einkaufspreis & $\begin{array}{l}\text { Eigene Aufwen- } \\
\text { dungen }\end{array}$ & Veräußerungspreis & $\begin{array}{l}\text { Gewinn } \\
\text { Verlust }\end{array}$ \\
\hline A & 0 & $8.000 \mathrm{WE}$ & $10.000 \mathrm{WE}$ & $+2.000 \mathrm{WE}$ \\
\hline B & $10.000 \mathrm{WE}$ & $4.000 \mathrm{WE}$ & 16.000 WE & $+2.000 \mathrm{WE}$ \\
\hline $\begin{array}{l}\text { C1 und } \\
\text { C2 }\end{array}$ & $2 \times 8.000 \mathrm{WE}$ & $4.000 \mathrm{WE}$ & $22.000 \mathrm{WE}$ & $+2.000 \mathrm{WE}$ \\
\hline $\begin{array}{ll}\text { D1 bis } \\
\text { D10 }\end{array}$ & $10 \times 2.200 \mathrm{WE}$ & $4.000 \mathrm{WE}$ & 28.000 WE & $+2.000 \mathrm{WE}$ \\
\hline $\begin{array}{l}\text { E1 bis } \\
\text { E1.000 }\end{array}$ & $1.000 \times 28 \mathrm{WE}$ & $0 \mathrm{WE}$ & $0 \mathrm{WE}$ & $-28.000 \mathrm{WE}$ \\
\hline
\end{tabular}

\section{Auswirkungen aufFallbeispiel 2}

Ähnlich wäre die Situation in Fall 2: Hier würde der Vergütungsanspruch des Vermieters V um dessen ersparte Aufwendungen gemindert. Die Pianistin $\mathrm{P}$ würde - wie auch in einer Situation, in der keine Leistungsstörung vorliegt - von dem Umstand profitieren, dass sie keine Fixkosten hat; sie erspart keine Aufwendungen, sodass ihr Vergütungsanspruch ungemindert bleibt. Der Veranstalter X schließlich müsste die reduzierten Vergütungsansprüche von $\mathrm{V}$ und $\mathrm{P}$ erfüllen und sich seine ersparten Aufwen- 
dungen anrechnen lassen. K1 und K1.000 müssten schließlich für das Konzert, das sie nie besuchen konnten, $45 \mathrm{WE}$ (statt der regulären $60 \mathrm{WE}$ ) bezahlen.

\begin{tabular}{|l|l|l|l|}
\hline & Eigene Aufwendungen & Vergütung & Gewinn / Verlust \\
\hline Vermieter V & $10.000 \mathrm{WE}$ & $20.000 \mathrm{WE}$ & $+10.000 \mathrm{WE}$ \\
\hline Pianistin P & $0 \mathrm{WE}$ & $10.000 \mathrm{WE}$ & $+10.000 \mathrm{WE}$ \\
\hline Veranstalter X & $\begin{array}{l}30.000 \mathrm{WE} \text { extern }+5.000 \mathrm{WE} \\
\text { intern }\end{array}$ & $45.000 \mathrm{WE}$ & $+10.000 \mathrm{WE}$ \\
\hline K1 bis K1.000 & $1.000 \times 45 \mathrm{WE}$ & $0 \mathrm{WE}$ & $-45.000 \mathrm{WE}$ \\
\hline
\end{tabular}

Dabei ist das entscheidende Argument für die Lösung nicht, dass ein Konzertbesuch „Luxus" ist und sich K1 bis K.1000 regelmäßig den Verlust einer Karte werden leisten können (es mögen sich andere Beispiele bilden lassen, in denen das nicht ohne weiteres der Fall ist und eine etwaige Härtefallregelung greifen würde). Entscheidend ist vielmehr, dass die Liquiditätseinbuße von $45 \mathrm{WE}$ so gering ist (verglichen zu den Liquiditätseinbußen, die nach geltendem Recht die Schuldner zu tragen haben), dass die Gefahr der Zahlungsunfähigkeit und damit der Insolvenz für alle Beteiligten abgewendet wäre. Das Eingreifen einer Härtefallregelung bei einigen wenigen Konzertbesuchern würde - sofern sie als Ausnahme mit strengen Voraussetzungen konzipiert wird - an dem grundsätzlichen Effekt der Regelung nichts ändern.

\section{Ein letzter Blick auf Fallbeispiel 3}

Mit Blick auf die Schlechtleistung wurde bereits darauf hingewiesen, dass sie in keinerlei Zusammenhang mit der Corona-Krise steht. Allenfalls kann sich die Corona-Krise auf die Möglichkeiten des Schuldners auswirken, einen Mangel zu beheben, etwa wenn die Nacherfüllung (vorübergehend) unmöglich wird. In diesem Fall ist de lege lata ein an sich behebbarer Mangel zu behandeln wie ein unbehebbarer Mangel.

Eine andere Frage ist, ob in Übertragung der Ausführungen zum Erhalt des Gegenleistungsanspruchs und zur Suspendierung des Rücktrittsrechts auch in Fällen der Schlechtleistung das Recht des Gläubigers zum Rücktritt (und entsprechend: zur Minderung) suspendiert werden sollte. Die Frage ist grundsätzlich zu verneinen, weil ein die Gewährleistungsrechte auslösender Sach- oder Rechtsmangel in seiner Entstehung von der Corona-Krise regelmäßig unabhängig ist. Handelt es sich um einen behebbaren Mangel, bleibt es daher bei der uneingeschränkten Nacherfüllungspflicht 
des Schuldners. Handelt es sich um einen Mangel, der nach seiner Natur unbehebbar ist, bleibt es dabei, dass ein Rücktritt, eine Minderung sowie die Geltendmachung eines Anspruchs auf Schadensersatz statt der Leistung ohne vorherige Fristsetzung möglich sind.

Etwas anderes gilt allerdings in den Fällen, in denen (allein) aufgrund der Auswirkungen der Corona-Pandemie die Behebung des Mangels unmöglich wird. Die oben skizzierte Behandlung an sich behebbarer Mängel als unbehebbar hat zur Folge, dass dem Schuldner das Recht zur (an sich möglichen) Nacherfüllung genommen wird und der Gläubiger an sich fristgebundene Gewährleistungsrechte ohne vorherige Fristsetzung ausüben kann. Zwar stehen das Rücktrittsrecht des Gläubigers sowie der Anspruch auf Schadensersatz statt der ganzen Leistung weiterhin unter dem Vorbehalt, dass der Mangel nicht unerheblich ist ( $\$ 323$ Abs. 5 Satz 2 bzw. $\$ 281$ Abs. 1 Satz 3 BGB). Auch bei erheblichen Mängeln erscheint es, soweit ihre Unbehebbarkeit ausschließlich auf den Auswirkungen der Corona-Pandemie beruht, aber aus denselben Gründen sachgerecht, den Gläubiger an den Vertrag gebunden zu halten, wie in Fällen, in den aufgrund der Pandemie von vornherein keine Leistung erfolgt ist. In diesen Fällen sind das Rücktrittsrecht des Gläubigers und - daran anknüpfend - das Recht zur Minderung somit zu suspendieren. Anderenfalls würde der Schuldner, der aufgrund der Corona-Pandemie von vornherein gar nicht leisten kann, besser stehen als ein Schuldner, der eine mangelhafte Sache leistet und dem lediglich die Nacherfüllung aufgrund der Corona-Pandemie nicht möglich ist. 